\title{
Ética profesional del docente universitario de psicología frente a un panorama multi-paradigmático
}

\author{
William Montgomery Urday* \\ Universidad Nacional Mayor de San Marcos, Lima, Perú \\ https://orcid.org/0000-0003-23485939.
}

Recibido: 13/09/2019 Revisado: 26/03/2020 Publicado: 20/05/2020

\begin{abstract}
Resumen
En esta comunicación se recuerda que la psicología es una ciencia multi-paradigmática en la cual, por lo mismo, se ha optado por una enseñanza universitaria de tipo ecléctico para reflejar todas las alternativas. Sin embargo, a veces en el desempeño práctico, la postura teórica personal de cada docente puede inclinarle a que seleccione y exponga de forma poco ética temáticas incongruentes con el equilibrio deseado, a la hora de presentar a sus alumnos la baraja completa de opciones de conceptualización y aplicaciones características de cada una de ellas. Este sesgo puede cometerse de varias maneras: privilegiando la mención de una alternativa sobre otras o contrastarlas desventajosamente; prohibiendo o sancionando respuestas que no considera válidas desde su punto de vista; o desatendiendo u omitiendo aclaraciones sobre lo discutido en clase. Se remarca que el correcto desempeño de la labor docente requiere una adecuada transmisión de conocimientos actualizados acerca de cualquier temática o dominio que se enseñe, por encima de las preferencias personales y profesionales.
\end{abstract}

Palabras clave: Psicología, ética profesional, paradigma, teoría, enseñanza.

\section{Professional ethics of the university teacher of psychology concerning a multi-paradigmatic panorama}

\begin{abstract}
This study aims to remind that psychology is a multi-paradigmatic science, which is why teaching psychology in higher education is eclectic in nature in order to reflect on the alternatives available in this respect. However, when it comes to practical performance, the personal theoretical orientation of each professor may lead him or her to opt for and present in an unethical manner topics that are not consistent with the desired outcomes of introducing students with the array of conceptualization options and their specific applications. This bias can occur when professors favor an alternative at the expense of others or make a biased comparison between them, forbid or penalize answers that they consider invalid, or neglect and avoid clarifying topics covered in class. This study highlights that the transmission of updated knowledge on a specific topic or domain is key for adequate faculty performance, which should rise above personal or professional preferences.
\end{abstract}

Keywords: Psychology, professional ethics, paradigm, theory, teaching. 


\title{
Ética profissional do professor de psicologia universitária versus uma paisagem multiparadigmal
}

\begin{abstract}
Resumo
Em esta comunicação recorda-se que a psicologia é uma ciência multiparadigmática. Por este motivo, optou-se por um ensino universitário de tipo eclético que reflita todas as alternativas. Porém, às vezes, no desempenho prático, a postura teórica pessoal de cada docente pode incliná-lo a selecionar e expor de maneira pouco ética temáticas incongruentes com o equilíbrio desejado no momento de apresentar aos seus alunos um leque completo de opções de conceptualização e das aplicações características de cada uma de elas. Diante de este viés, é possível agir de várias maneiras: privilegiando a menção de uma alternativa sobre as outras ou contrastando-as de maneira desigual; proibindo ou punindo respostas que não considera válidas desde seu ponto de vista; desatendendo ou omitindo esclarecimentos sobre o que foi discutido na sala de aula. Salienta-se que o desempenho correto do trabalho do docente precisa de uma transmissão de conhecimentos atualizados sobre qualquer temática ou domínio que ensine, acima de quaisquer preferências pessoais e profissionais.
\end{abstract}

Palavras-chave: Psicologia, ética profissional, paradigma, teoria, ensino

Citar como:

Montgomery, W. (2019). Ética profesional del docente universitario de psicología frente a un panorama multi-paradigmatico. Revista Digital de Investigación en Docencia Universitaria, 14(1), e1033. https://doi.org/10.19083/ridu.2020.1033

Fis. profesor universitario organiza y media la estructuración del conocimiento en sus alumnos, y para llevar a cabo dicha labor debe contar con algunas capacidades profesionales que pasan por el dominio teórico de lo que enseña; el uso de estrategias de transmisión de contenidos lectivos; y el ejercicio de valores y actitudes que apuntalen su manejo docente. En este último punto se halla la ética del trabajo académico, que dota a sus tareas de un sentido auténticamente científico cuando, como señala Ander-Egg (1995), se constituye "no como forma de ser para cuando "se hace ciencia", sino como actitud vital en todas las circunstancias y momentos de la vida [...] lo que llamamos la actitud científica como estilo de vida" (p. 121). Ella implica, entre otras cosas, ser ético y escrupuloso con los contenidos que se transmiten sin mezclarlos con opiniones personales que no se expliciten como tales. Max Weber se refirió a este tema de una manera tajante en su famosa disertación de 1919 sobre la relación entre política y ciencia en el salón de clase, afirmando que:

[...] no está en las aulas el puesto del demagogo o del profeta [...] Me parece de una absoluta falta de responsabilidad que el profesor aproveche estas circunstancias para marcar a los estudiantes con sus propias opiniones políticas, en lugar de limitarse a cumplir su misión específica, que es la de serles útil con sus conocimientos y con su experiencia científica. (Weber, 1979, p. 213)

El reclamo de Weber se refiere a la política, pero, con la misma lógica, también es válido para la emisión de cualquier otra opinión personal del docente que se quiera hacer pasar como si fuera ex cátedra sobre la discusión de un tema o los contenidos de un curso. De hecho, hay documentos de alto nivel que procuran prevenir este tipo de prácticas, como por ejemplo en nuestro medio el apartado sobre deberes del docente (artículo 87) de la Ley Universitaria 30220 vigente en el Perú, que remarca la necesidad de: "Ejercer la docencia con rigurosidad académica, respeto a la propiedad intelectual, ética profesional, independencia y apertura conceptual e ideológica" (Congreso de la República, 9 de julio de 2014; p. 527224).

Lo cierto para la docencia universitaria en general, lo es también para sus ejercicios de enseñanza en las diferentes carreras, como, en este caso, de la psicología. Sin embargo, debe tenerse en cuenta que las características particulares de esta disci- 
plina hacen más complicado seguir el mandato implícito de evitar la intromisión de opiniones personales no sustentadas por la evidencia, por las razones explicadas en los siguientes párrafos.

No es un secreto que en la psicología existen múltiples opciones conceptuales. Desde su punto de partida moderno, esa diversidad se manifestó en la formulación y reformulación continuada de diferentes objetos de estudio, teorías, modelos, métodos y otros contenidos relevantes (Gondra, 2003), dando lugar a una larga y compleja historia de competición paradigmática; así como, consecuentemente, a una imagen de fragmentación y desorden (cuando no de confusión) para el observador de su devenir y estado actual (Arana, Meilán, \& Pérez, 2006; López \& Fernández, 2015). Frente a dicha realidad el tema de su enseñanza académica en términos integrales se convierte en un grave problema.

A este respecto, en un intento de simplificación sintética, se ha clasificado la psicología en general de acuerdo con esquemas dicotómicos, como por ejemplo el de la existencia de dos culturas enfrentadas en la misma disciplina: cientificista y comprensiva (Cornejo, 2005), cada cual con propósitos e intereses distintos: experimental-correlacional, por un lado, y hermenéutico-fenomenológico por otro. Esta división parece congeniar con la establecida por el clásico trabajo de Coan (1979) sobre las dimensiones básicas subyacentes como tendencias en la teoría psicológica: restrictiva (similar a la experimental-correlacional) adversus fluida (similar a la hermenéutico-fenomenológica) (véase Montgomery, 2006, para una revisión). También se ha procurado reducir el ámbito total de opciones a unas pocas, como en el caso de Ardila (2003), para quien el panorama comprende los enfoques neoconductista, neopsicoanalítico, humanista e histórico-cultural.

De cualquier forma, la solución tradicional adoptada para la enseñanza de la psicología ha sido el eclecticismo. Vale decir, articular en un plan de estudios un conjunto de asignaturas cuyo abordaje dé supuesta cuenta de todos, o de la mayoría, de los enfoques considerados vigentes en la disciplina. Los referentes para este tipo de asunción han sido las numerosas introducciones a la psicología general (por ejemplo los conocidos manuales de Feldman, 2014, y de Morris \& Maisto,
2019; así como sus numerosos émulos en los países de habla hispana), que presentan esos tópicos en términos que podrían denominarse de multiplicidad compatible de paradigmas y contenidos, minimizando las diferencias reflejadas en los distintos compromisos ontológicos de cada corriente, y privilegiando la exposición de las operaciones empíricas y prácticas supuestamente comunes que se formulan con base en la demanda social ${ }^{1}$.

La contradicción de semejante estrategia ha sido y es que, si bien los currículos pueden ser eclécticos, en cambio la inmensa mayoría de profesores que viabilizan su cumplimiento en clase de aula no lo son, pues ellos tienen una postura teórica personal (inevitable en una disciplina multiparadigmática) aunque a veces no la expliciten suficientemente. He aquí lo que puede originar el problema que es materia de la presente comunicación: un problema de posible sesgo en la transmisión de conocimientos pertinentes por parte del docente.

\section{Problema de sesgo del docente de psicología}

La definición de sesgo, en el diccionario de la Asociación Psicológica Americana (2010), implica "parcialidad: la inclinación o predisposición a favor o en contra de algo. Véase también prejuicio" (p. 463). En el asunto que se está tratando en este artículo, se emplea para designar una tendencia consciente o inconsciente del docente universitario a desbalancear su enseñanza de ciertos cursos (o parte de ellos), con base en inclinaciones paradigmáticas y teóricas personales.

Abundando sobre esto: por un lado, el docente que se hace cargo de una asignatura diseñada para presentar una perspectiva panorámica de alguna área general o introductoria de la psicología, frecuentemente puede llegar rodeado de un nimbo de imparcialidad que hace suponer a sus alumnos que las exposiciones que lleva a cabo acerca de los temas considerados en su sílabo, son fieles y abarcadoras de toda la baraja de enfoques y teorías que existen. Pero, por otro lado, en la praxis puede mostrar que tanto la selección que

1 Solo para mencionar algunos de esos manuales en nuestro país: Uculmana (1986), Meza (2002), Braun (2009) y Frisancho (2011). 
el docente hace de sus temas, como su exposición misma y las valoraciones críticas ligadas a cada contenido, están plagadas de sesgos relacionados con sus opciones personales y profesionales. Vale decir, no presenta el panorama real ni siquiera de una manera aproximada, sino su versión del mismo de manera que podría calificarse por lo menos como poco responsable. Eso podría considerarse asimismo profesionalmente poco ético, a pesar de lo cual suele minimizarse o ignorarse como si fuera algo natural o inevitable, o propio simplemente de la falta de suficiencia para enseñar. Aquí, por cierto, no se cuestiona la atribución formal que tiene cada docente para organizar con libertad su curso. Eso está bien y es deseable. Lo cuestionable éticamente es que al hacerlo no tenga el cuidado, por decir lo menos, de revisar o actualizar la literatura que da cuenta del tema que trata, a veces sobre la base de presuposiciones acerca de que tal o cual fuente paradigmática de la que proviene es incorrecta o está desfasada, o, cuando menos, dejar claro ante sus alumnos desde qué perspectiva paradigmática hace su selección y exposición de temas. Debería estar sobreentendido que un buen profesional es escrupuloso e imparcial per se respecto a la enseñanza del conocimiento que pretende impartir, lo cual no quiere decir que no pueda dar su opinión en clase después de haber proporcionado a los estudiantes toda la información posible, sin sesgos excesivos ni suficiencia pomposa sobre la temática transmitida.

Como dicen Guzmán y Guzmán (2016), refiriéndose a la realidad mexicana, pero con un sentido universal:

Tal parece que los psicólogos hemos asumido que no necesitamos prepararnos para enseñar psicología pues lo que recibimos en nuestra formación nos habilita para enseñar y evaluar adecuadamente [...] Decimos lo anterior porque en una búsqueda bibliográfica en las principales revistas psicológicas y educativas de nuestro país no se encontraron trabajos o investigaciones sobre experiencias reportadas de formación docente en psicología. (p. 8)

Lo corriente es que el profesor de una asignatura tenga su estrategia didáctica a partir de sus propios conocimientos, seleccionando aquellos contenidos que juzga pertinentes, enfatizando temáticas y desechando (o eludiendo) otras. En tal sentido, si, como sucede frecuentemente, se hace cargo de un dominio teórico o empírico de tipo panorámico (digamos Psicología General, Historia de la Psicología, Psicología Educativa, Psicología Clínica, Psicología del Lenguaje, Epistemología, Psicología de la Personalidad, Psicoterapia y otros similares), en el peor de los casos puede tender a elaborarlo de manera tan parcial que llegue a ser subjetiva e incluso prejuiciosa, ignorando, o, peor, malinterpretando o tergiversando la contribución de enfoques que no son el suyo, y magnificando éste a su vez, presentándole a los alumnos una imagen distorsionada de lo que es el dominio en mención.

Lo cierto es que muchos docentes haciendo lo mismo a favor de un enfoque eventualmente mayoritario en militancia, producen un efecto amplificado de lo que se ha llamado "efecto San Mateo" (como se citó en Bunge, 2019): “porque a cualquiera que tiene, le será dado, y tendrá más; pero al que no tiene, aun lo que tiene le será quitado" (versículo 13 del capítulo 19 del Evangelio atribuido a San Mateo). O sea, que le atribuyan todos los méritos al paradigma o teoría momentáneamente dominante en perjuicio de paradigmas o teorías menos populares o influyentes. Es obvio que en ciencia la democracia (u oclocracia) no puede ser un criterio de referencia para formular juicios sobre la adecuación o inadecuación de informes sobre la realidad.

La objeción que algún partidario del sesgo podría formular a lo expresado en el párrafo anterior sería que, si una mayoría de miembros de la comunidad profesional está de acuerdo con un enfoque en particular, debe ser porque éste es mejor o soluciona más problemas al estilo de la argumentación kuhnniana sobre las revoluciones paradigmáticas, y por lo tanto resulta casi natural que se prefiera más. Esta clase de razonamientos, muy manidos en la disciplina durante las décadas del 60 al 80 del siglo XX, ha perdido fuerza desde hace años (por ejemplo, ver las refutaciones hechas por los historiadores Leahey, 1992; Goodwin, 2015). Salvo casos muy específicos, lo que suele predominar en la psicología no es un paradigma, sino que se trata, al menos por ahora, de una coexistencia o competición multiparadigmática. 


\section{La influencia de los libros de texto sesgados}

A pesar del descrédito de la leyenda de un paradigma que sustituye a otros menos capaces, hay muchos colegas que siguen opinando en ese sentido, y ciertamente, uno de los soportes más efectivos de esa corriente de opinión es la narrativa ofrecida por ciertos manuales introductorios a la psicología. Y es que, con frecuencia, el exceso de exigencias a las que se le somete al docente universitario parece obligar a algunos a plantear sus contenidos de enseñanza únicamente con base en la lectura de unos pocos libros generales, en vez de revisar a consciencia toda la literatura posible sobre los temas en que pretende ser experto ante los estudiantes.

Ciertamente, la práctica de presentar obras sesgadas a favor de una orientación en particular como si fueran una auténtica revisión del campo psicológico en cualquiera de sus áreas de estudio, ha sido muy frecuente, sirviendo de inadecuado modelo a los docentes universitarios. Entre muchas otras, las introducciones bibliográficas aludidas en la primera sección de este artículo son un buen ejemplo de prácticas sesgadas negativas que, en cierto modo, canonizan otras extendidas a la enseñanza de aula. A saber, si uno revisa el libro de Morris y Maisto (2019), se percata que, ya desde las primeras páginas, hay un sesgo establecido respecto, entre otras cosas, al entusiasta apoyo que los autores le dan a uno de los enfoques (en este caso el cognoscitivo) en detrimento del considerado competidor principal (en este caso el conductista), al punto de declarar expeditamente: "En contraste con el conductismo, los psicólogos cognoscitivos creen que los procesos mentales pueden y deben estudiarse científicamente" ( $p$. 15). Eso no solo es una notable imprecisión basada en un popular prejuicio, no sostenible argumentalmente solo revisando un poco la literatura conductual - lo que pone en duda la idoneidad profesional de quienes la deslizan tan ligeramente-, sino una falta de honestidad inesperada en aquellos que, emprendiendo el trabajo de hacer una presentación general de la disciplina, no preservan un mínimo de imparcialidad. Lo correcto, en todo caso, respecto a cualquier obra que pretenda dar una visión panorámica de una disciplina, sería que su título refleje de entrada la orientación particular (muy legítima por cierto) de los auto- res, en vez de aparentar estar por encima de todas las teorías. Pero no, el título de dicho libro parece ser totalmente aséptico: Understanding Psychology (Introducción a la Psicología en su traducción hispana).

Algo que debe entenderse es que cualquier concepción explicativa individual, además de coadyuvar a disponer cómo relacionarse con los problemas prácticos, responde a su vez a un planteamiento mayor que lo enmarca. El psicólogo que funge de profesor de psicología -igual que el autor de un libro o artículo de divulgación- no sale de una burbuja que lo ha preservado de toda contaminación teórica. Él determina de forma idiosincrática lo que hace o dice, promueve o retroalimenta en su clase, en función a los criterios que delimitan su propia perspectiva: sus ejemplares, es decir aquellos que le han llevado a establecer sus abstracciones acerca del reconocimiento de los eventos que parecen importantes en las diversas temáticas.

Como ya se ha sugerido, eso en sí mismo no está mal, ya que supone libertad de pensamiento y de cátedra. Lo que está mal es que el docente lo haga bajo la suposición consciente o no de que su perspectiva es la única correcta y que por ello debe transmitir ese sentimiento valorativo a sus alumnos, administrando además las fuentes que les da a conocer sobre el punto de modo que certifiquen o confirmen aquello que sostiene. Lo cierto es que aunque uno puede pensar de manera honesta que lo que cree o sabe es mejor que lo que otros creen o saben, y puede argumentarlo bien, la labor docente no permite éticamente ser parcial en ningún caso debido a la imagen de autoridad que ostenta el docente frente a su joven alumnado (recordar nuevamente la disquisición de Weber, 1979; ut supra), en ejercicio del poder para influenciar de una manera muy profunda sobre las creencias y actitudes de aquel, con el peligro de que éstas terminen transformándose en prejuicios y estereotipos de aceptación o rechazo muy superficiales hacia corrientes de la psicología que no sean de su agrado.

La manera cómo la postura teórica enfática del profesor de una asignatura puede causar impacto sobre los alumnos varía de acuerdo a ciertas modalidades en relación con su manera de encarar las interacciones con ellos, a saber: las prácticas 
mediadoras de modelar o instruir sobre algún tipo de contenido, o acerca de los requerimientos a cumplir previos a él; de señalar una opción sobre otras o contrastarlas; de auspiciar o disponer condiciones para que se dé una transferencia de aprendizaje o de instruir sobre las demandas sociales que aquella debe satisfacer; de prohibir o de sancionar respuestas mediante consecuencias concretas; y de desatender u omitir alguna explicitación sobre lo discutido en clase. En este amplio sentido la función del docente es el de un constructor de contextos dentro del aula (García \& Vargas, 2008), que, a través de sus estrategias de enseñanza, modela tanto actitudes como respuestas puntuales en sus alumnos respecto a las opciones que existen en el universo teórico de la psicología.

Se podrían dar multitud de ejemplos informales de cómo en clases, en charlas, conferencias, publicaciones y otros tipos de exposiciones orales y escritas, se dicen y se repiten muchas cosas sin sustento bajo el manto de una supuesta imparcialidad o supra-conocimiento, al no compartir cosmovisión con ciertos paradigmas o teorías que son objeto de la crítica, simplificación o tergiversación del expositor. Baste reseñar dos: un alumno oyó en clase a su profesor reseñar entusiastamente el panorama de las teorías según él vigentes en el plano del lenguaje y pensamiento. Al requerir éste preguntas acerca de lo dicho, le fue hecha una que inquiría sobre la ausencia de teorías $X$ e $Y^{2}$ en su presentación. Su respuesta, rápida y cortante, fue: “Es que esas corrientes no tienen teorías sobre lenguaje ni sobre pensamiento", confundiendo el hecho de que dichas corrientes obvien etiquetar sus estudios sobre temas así con el de que no se ocupen de ellos. Otro ejemplo va con los numerosos manuales sobre personalidad que circulan y se utilizan en la asignatura de Teorías de la Personalidad (entre otras). Si usando esos manuales como libros de texto el profesor de dicho curso se tuviera que atener solo a lo que muestran, se tendría que limitar a exponer sobre algunos teóricos psicodinámicos, otros tantos humanistas, un conductista anti-personalidad (Skinner), un antiguo constructivista, varios facto-

2 Se usan $X$ e $Y$ para evitar menciones de teorías con nombre propio, a fin de no contaminar la argumentación con referencias de parte. rialistas y otros de aprendizaje social, punto. Pero ¿dónde quedan otros teóricos conductistas más recientes que sí hablan de personalidad? ¿dónde los cognitivo-constructivistas actuales? ¿dónde los teóricos marxistas? Simplemente son ignorados. Lo mismo, con las variaciones del caso, se ve en otras materias. El hecho es que la mayoría de docentes se atienen a ese recorte como si fuera natural.

En realidad, basta revisar con cierta profundidad la literatura de cualquier corriente para encontrar que sí tiene concepciones teóricas sobre pensamiento, lenguaje y personalidad, aunque en libros de texto que pretendan hacer revisión panorámica de dichos rubros no aparezcan. Solo para ilustrar lo dicho se pueden citar textos de revisión sobre la temática de la personalidad en autores conductistas (Santacreu, Hernández, Adarraga, \& Márquez, 2002), marxistas (González Rey, 2002), y constructivistas (Balbi, 2004) que no son incluidos ni mencionados en los libros de texto tradicionales. Algo parecido ocurre en otras temáticas, pero sería largo y marginal a la presente argumentación citar más materiales en relación con ello.

Es deber ético y deontológico del profesor de una materia no basarse solo en revisiones sumarias hechas por otros, sino en profundizar por sí mismo sobre lo que va a enseñar teniendo en cuenta la multiplicidad paradigmática de la disciplina. Para concretar este propósito nada mejor que ir a las fuentes de aquello que se expone en clase, evitando los manuales generales para concentrarse en obras o artículos básicos de autores de la propia corriente que desarrolla conceptos, métodos o investigaciones sobre tal o cual tema de tratamiento.

\section{¿Posible solución?}

Teniendo en cuenta lo dicho en anteriores apartados, hace años, con motivo de una reforma curricular en la Universidad Nacional Mayor de San Marcos (UNMSM), asumiendo la multiplicidad de concepciones en la psicología, el autor de este artículo propuso dos cosas:

a) Que aquellos cursos del plan de estudios que presentaran un panorama global de alguna área de la psicología (como los señalados 
más arriba en la primera sección: Psicología Educativa, Psicología Clínica, Psicología del Lenguaje, Epistemología, Psicología de la Personalidad, Psicoterapia, entre otros), fueran dictados como asignaturas colegiadas por grupos de docentes de diferentes orientaciones psicológicas. Esto con el propósito de auspiciar exposiciones actualizadas sobre esos temas, por parte de quienes realmente conocen - por provenir de, y trabajar en una orientación particular - el campo desde la perspectiva de su preferencia y ejercicio profesional.

b) Que, dentro de dicho plan, se incluyeran cursos de introducción teórica y práctica a los diferentes paradigmas de la psicología (Psicología Conductual y Técnicas de Intervención Conductual; Psicología Cognitiva y Técnicas de Intervención Cognitiva; Psicología Dinámica y Técnicas de Intervención Dinámica; Psicología Humanista y Técnicas de Intervención Humanista; Psicología Dialéctica y Técnicas de Intervención Dialéctica), para proporcionar al estudiantado revisiones integrales comprometidas con diferentes cosmovisiones en términos filosóficos, teóricos, metodológicos, investigativos, tecnológicos y aplicativos, expuestas por docentes del rubro.

El objetivo era presentar múltiples opciones para que los mismos alumnos compararan y eligieran alguna de ellas como motivación para su actividad lectiva. Infortunadamente, la primera propuesta no pudo aplicarse por problemas relativos a los horarios de trabajo de los docentes, pero la segunda sí se aplicó parcialmente, incluyendo cuatro asignaturas dedicadas a la exposición global de paradigmas Psicología y Técnicas de Intervención Conductuales; Psicología y Técnicas de Intervención Cognitivas; Psicología y Técnicas de Intervención Dinámicas; y Psicología y Técnicas de Intervención Humanistas), actualmente presentes en el plan de estudios de las dos escuelas de la Facultad de Psicología de la mencionada universidad. En la actualidad, las sumillas de todos ellos exigen la presentación integral de las producciones relevantes de la cosmovisión psicológica en los términos que ya se han mencionado.
No es la perfección, pero en otras instituciones educativas superiores nacionales, que se sepa, no ha habido intentos tan radicales como el señalado para evitar sesgos teóricos o compensarlos.

Además de lo señalado, obviamente haría falta una mayor atención a los problemas de la formación respecto a las competencias necesarias en la enseñanza docente, así como de sus aspectos ético-profesionales. Hablar de ello en vez de asumir que no existe.

\section{Conclusión}

Asumir una actitud científica como estilo de vida implica también ser ético y escrupuloso con los contenidos que se transmiten en la tarea docente. El hecho de tener una postura paradigmática y teórica particular dentro de un universo que se sabe multiparadigmático, no exime al profesor de una asignatura de procurar ser fiel selector y expositor de los contenidos pertinentes de cada opción psicológica, sobre todo en cursos que asumen doctrinariamente una neutralidad formal acerca de los temas tratados, con el fin de que sus estudiantes contacten con auténticas versiones de todas las alternativas.

Para prevenir posibles sesgos paradigmáticos poco éticos en semejante labor se requiere, desde la perspectiva presentada aquí, por un lado, de ajustar la práctica expositiva a criterios lo más exhaustivos posibles en la búsqueda de información a ser desarrollada sobre cada corriente o enfoque en asignaturas panorámicas de la disciplina (lo cual incluye si es posible, como se ha dicho, encomendarla a gente identificada con cada enfoque para su dictado); y, por otro, sustituir el uso de introducciones o panoramas generales - falsamente neutrales de acuerdo con el razonamiento que guía esta propuesta-, por libros o artículos básicos elaborados por autores relevantes de cada opción.

Ello iría en beneficio del alumnado, y de promociones futuras de psicólogos mejor informados y comprometidos con el desarrollo de su disciplina. En otras palabras, capaces de entender distintas perspectivas además de la propia y asumir tareas de investigación cuya coherencia teórica y práctica sea efectiva. 


\section{Referencias}

Ander-Egg, E. (1995). Técnicas de investigación social. Buenos Aires, Argentina: Lumen.

Asociación Psicológica Americana (2010). Diccionario conciso de psicología. México: El Manual Moderno.

Arana, J. M, Meilán, J. J. \& Pérez, E. (2006). El concepto de psicología. Entre la diversidad conceptual y la conveniencia de unificación. Apreciaciones desde la epistemología. Revista Intercontinental de Psicología y Educación, 8(1), 111-142.

Ardila, R. (2003). La necesidad de unificar la psicología. El paradigma de la síntesis experimental del comportamiento. Revista Colombiana de Psicología, 12, 28-37.

Balbi, J. (2004). La mente narrativa: Hacia una concepción posracionalista de la identidad personal. Buenos Aires, Argentina: Paidós.

Braun, R. (2009). ¿Qué soy yo? Una introducción a la filosofía de la mente y de la psicología. Lima, Perú: Fondo Editorial de la Universidad de Lima.

Bunge, M. (2019). El efecto San Mateo. Polis [En línea], 2 । 2002, http://journals.openedition.org/polis/8033

Coan, R. W. (1979). Psychologists: Personal and theoretical pathways. New York, USA: Irvington.

Congreso de la República (9 de julio de 2014). Ley Universitaria 30220. Diario Oficial El Peruano, pp. 527211527233. Recuperado de: https://www.sunedu.gob. pe/wp-content/uploads/2017/04/Ley-universitaria-30220.pdf

Cornejo, C. (2005). Las dos culturas de/en la psicología. Revista de Psicología de la Universidad de Chile, 14(2), 189-208. https://doi.org/10.5354/07190581.2005.17432

Feldman, R. S. (2014). Understanding psychology (12th Edition). New York, USA: McGraw-Hill.

Frisancho, A. (2011). Psicología general. Manual de prácticas y ejercicios. Lima, Perú: Universidad Nacional Mayor de San Marcos.
García, M. \& Vargas, P. (2008). Hacia la formación del psicólogo por competencias. En C. Carpio (Coord.). Competencias profesionales y científicas del psicólogo. Investigación, experiencias y propuestas (pp. 75 . 102). México: Universidad Autónoma de México.

Gondra, J. M. (coord.) (2003). La psicología moderna: Textos básicos para su génesis y desarrollo histórico. Bilbao, España; Desclée de Brouwer.

González Rey, F. (2002). Sujeto y subjetividad. Una aproximación histórico-cultural. México: Thomson.

Goodwin, C. J. (2015). A History of Modern Psychology (Fifht edition). New jersey, USA: John Wiley \& Sons.

Guzmán, J. C. \& Guzmán, M. (2016). Estrategias y métodos para enseñar contenidos psicológicos. México: Universidad Nacional Autónoma de México.

López, M. \& Fernández, J. (2015). Análisis del caos interparadigmático e intraparadigmático en psicología. Conductitlán, 2(1) 5-26.

Leahey, T.H. (1992). The mythical revolution of american psychology. American Psychologist, 47, 308-318. https://doi.org/10.1037/0003-066X.47.2.308

Meza, A. (2002). Introducción a la psicología. Lima, Perú: Universidad Ricardo Palma.

Montgomery, W. (2006). Estado actual del problema de la unificación paradigmática en psicología. En A. Quintana y W. Montgomery (Eds.). Psicología: Tópicos de actualidad (pp. 9-24). Lima, Perú: Universidad Nacional Mayor de San Marcos.

Morris, Ch. G. \& Maisto, A. A. (2019). Understanding psichology (Twelfth Edition). London, England: Pearson Education.

Santacreu, J.; Hernández, J.M.; Adarraga, P. \& Márquez, M.O (2002). La personalidad en el marco de una teoría del comportamiento humano. Madrid, España: $\mathrm{Pi}$ rámide.

Uculmana, Ch. (1986). Psicología general. Lima, Perú: Caribe.

Weber, M (1979). El político y el científico. Madrid, España: Alianza Editorial.

RIDU / Revista Digital de Investigación en Docencia Universitaria / e-ISSN: 2223-2516

๑) Los autores. Este artículo es publicado por la Revista Digital de Investigación en Docencia Universitaria del Área de Institutional Research and Effectiveness de la Dirección de Aseguramiento de la Calidad, Universidad Peruana de Ciencias Aplicadas. Este es un artículo de acceso abierto, distribuido bajo los términos de la LicenciaCreativeCommons Atribución-CompartirIgual 4.0 Internacional.( http://creativecommons.org/licenses/by-sa/4.0/), que permite el uso no comercial, distribución y reproducción en cualquier medio, siempre que la obra original sea debidamente citada. 